Proceedings of the 4th International Congress APMAS2014, April 24-27, 2014, Fethiye, Turkey

\title{
Thickness Dependent Magnetic Properties of Polycrystalline Nickel Thin Films
}

\author{
U. PArlak ${ }^{a, *}$, M.E. AKÖz ${ }^{b}$, S. TOKDEmir ÖZTÜrk ${ }^{b}$, M. ERKOvaN ${ }^{c}$ \\ ${ }^{a}$ Peter Grünberg Institut, Electronic Properties (PGI-6), Forschungszentrum Jülich, 52425, Jülich, Germany \\ ${ }^{b}$ Gebze Technical University, Department of Physics, 41400, Kocaeli, Turkey \\ ${ }^{c}$ Sakarya University, Department of Nanoscience and Nanoengineering, 54187, Sakarya, Turkey

\begin{abstract}
Magnetization directions of ferromagnetic ultra-thin films can be altered by increasing the thickness. The transition between in-plane and out-of-plane axes is induced by the competition among the magnetostatic, magnetocrystalline and magnetoelastic anisotropy energies. Such an effect has attracted more interest recently, due to the applications in magneto-optical recording technologies. In this study, we have investigated by magneto-optical Kerr effect the magnetization properties of magnetron sputtered nickel thin films.
\end{abstract}

DOI: 10.12693 /APhysPolA.127.995

PACS: $75.70 . \mathrm{Ak}, 75.30 . \mathrm{Gw}$

\section{Introduction}

Thin-film-based magnetic storage media are at the point of saturation nowadays. New generation products with perpendicular recording technology are considered as the way to reach higher densities $[1,2]$. Perpendicular recording, based on thin films, requires thin film magnetization to be explored.

Magnetic orientation of thin films strongly depends on competition between different magnetic anisotropies. The magnetization direction of a thin film experiences a transition from parallel to perpendicular orientation, this ia the so-called spin reorientation transition (SRT). In order to obtain SRT behavior, some physical properties could be manipulated such as composition and thickness of the film, as well as the type of the substrate.

The thickness dependent behavior of the magnetization is expressed by the equation below and is visualized in Fig. 1 (adopted from [3]).

$$
K_{\mathrm{eff}}=K_{\mathrm{u}}-2 \pi M_{\mathrm{s}}^{2}+K_{\mathrm{me}}+K_{\mathrm{s}} / t .
$$

The first and the second terms in Eq. 1 are the main components which compete for the determination of the magnetization direction. All terms could be expressed as magnetocrystalline anisotropy, shape anisotropy, magnetoelastic anisotropy and surface anisotropy, respectively.

Effective anisotropy constant is separated into two parts representing the volume and the surface anisotropy contributions. Therefore, these two components favor inplane magnetization where $K_{\text {eff }}<0$ and vice versa. With increasing thickness, MCA term dominates and magnetization switches to perpendicular direction at a critical thickness value. For thick films, the shape anisotropy is the dominating contribution, that determines the easy axis of the film.

*corresponding author; e-mail: u.parlak@fz-juelich.de

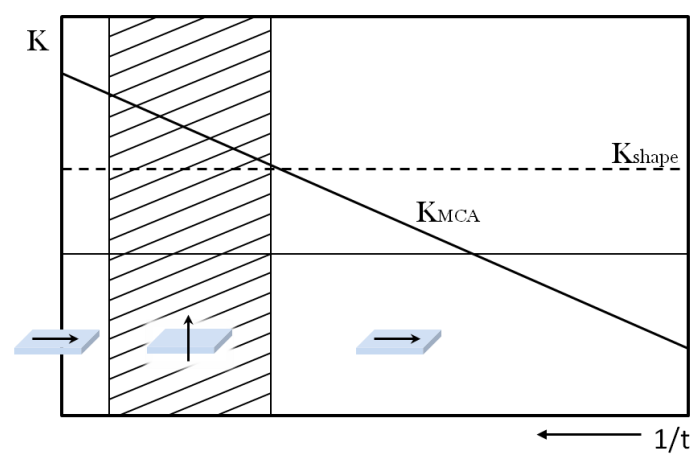

Fig. 1. Magnetization transition behavior of ferromagnetic thin films with respect to inverse film thickness.

$\mathrm{Ni}$ thin film is one of the possible candidates for observing such a behavior. Till present, Ni films were investigated on a number of different substrates. Schulz and Baberschke investigated SRT behavior of $\mathrm{Ni}$ on $\mathrm{Cu}(100)$ substrates for two different thicknesses [4]. Erkovan et al. reports that $\mathrm{Ni}$ films on $\mathrm{Cu}_{3} \mathrm{Au}$ exhibit SRT at a thickness of $8 \mathrm{ML}$ [5].

\section{Sample preparation}

Samples were fabricated at room temperature using magnetron sputtering system (UHV Sputtering System 200 , Bestec). Ni thin films were deposited on a naturally oxidized Si substrate after annealing process. High purity $\mathrm{Ni}$ target was sputtered at the Ar gas pressure of $1.2-1.4 \times 10^{-3}$ mbar and at sputter power of $30 \mathrm{~W}$. the substrate-target distance was kept constant at $65 \mathrm{~mm}$. Ni deposition rate was determined using XPS under the same deposition conditions, for the accurate thickness values.

In order to determine the deposition rate of $\mathrm{Ni}$, photoemission signals of substrate have been collected starting from as-annealed substrate to the $\mathrm{Ni}$ deposited substrate, for 70 seconds. Si 2 s peak was selected instead of the 
major peak of $\mathrm{Si}(\mathrm{Si} 2 \mathrm{p})$, in order to prevent the overlapping with $\mathrm{Ni} 3 \mathrm{~s}$ peak $(111 \mathrm{eV})$. On the other hand, Si 2 s and Si 2 p peaks both have the same atomic sensitivity factors (ASF). A polynomial background function was used to eliminate the background intensity. After dividing by ASF, the areas under the peaks were calculated using Voigt function, which is appropriate for $\mathrm{Si}$ (Fig. 2a). The intensities were converted to thickness values with the help of attenuation length formula,

$$
I=I_{0} \exp \left(-\frac{d}{\lambda}\right) \text {. }
$$

In order to calculate the inelastic mean free path values of Si 2s electrons within the Ni film, NIST Electron Inelastic-Mean-Free-Path Database (v1.2) was used and Tanuma-Powell-Penn (TPP) formula was selected [6]. The data were fitted linearly (Fig. 2b) and the calculated $\mathrm{Ni}$ deposition rate was $0.44 \AA / \mathrm{s}$. Four samples with $\mathrm{Ni}$ thickness of $12,14,24$ and $26 \AA$ were fabricated within certain amount of deposition time.
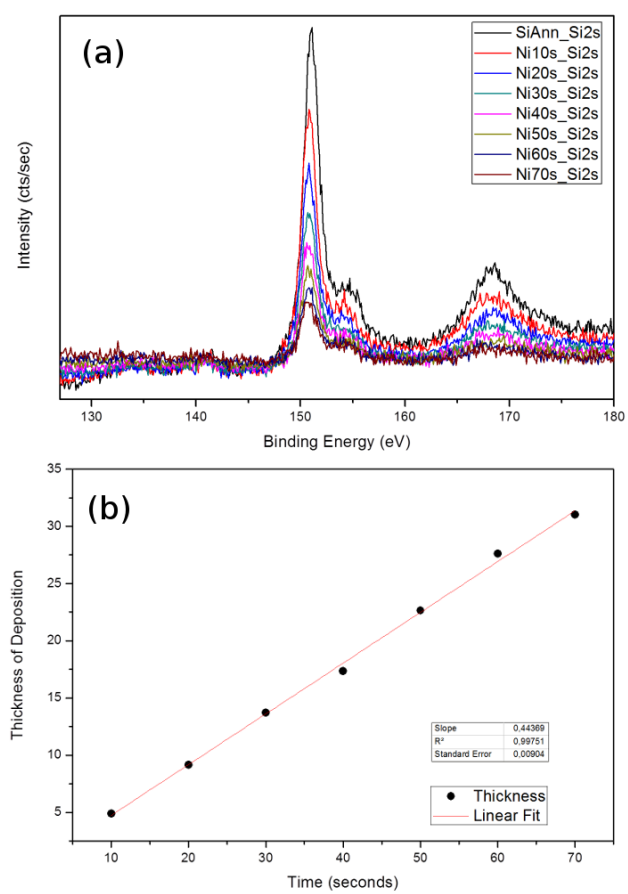

Fig. 2. a) XP spectra of as-annealed and Ni deposited substrate, measured between 10 and 70 seconds, b) linear fit of the thicknesses calculated from the XP spectra.

\section{Results}

Magnetic characterization of the samples has been performed by magneto-optical Kerr effect system (SmartMOKE Magnetometry System, Nanosan Ins.) at room temperature. After the alignment process, magnetic field of $1 \mathrm{~T}$ (maximum) was applied in L-MOKE configuration, in direction 0,45 and 90 degrees with respect to an arbitrary reference axis. Easy axes, obtained from L-MOKE, were selected and shown in Fig. 3. Subsequently, the P-MOKE measurements were carried out in the same conditions.
In Fig. 3, the left column shows the hysteretic behavior of the samples under field applied parallel to film plane. For FM layers of 12 and $14 \AA$, the hard axes lay parallel to film plane and the saturation magnetizations are reached at $2.5 \mathrm{kOe}$ and $7.5 \mathrm{kOe}$ respectively. On the other hand, thicker layers need lower applied fields for saturation, particularly 40 Oe. In contrast to the in-plane case, easy axes are observed for thinner layers, when the field is applied perpendicular to the film plane. As it can be clearly observed from the right column, spin reorientation transfer occurs between 14 and $24 \AA$ of Ni layers thickness. Saturation magnetizations of thicker layers for out-of-plane case are reached at $7.5 \mathrm{kOe}$.
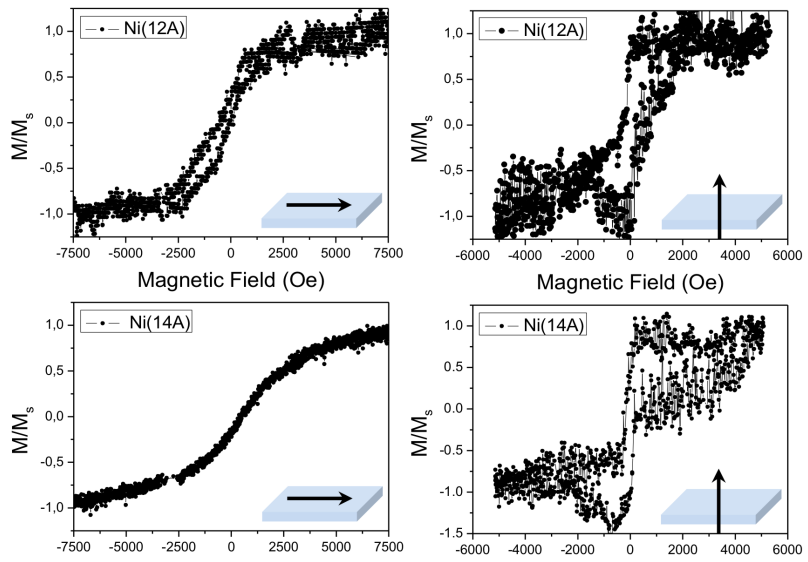

Magnetic Field (Oe) Magnetic Field $(\mathrm{Oe})$
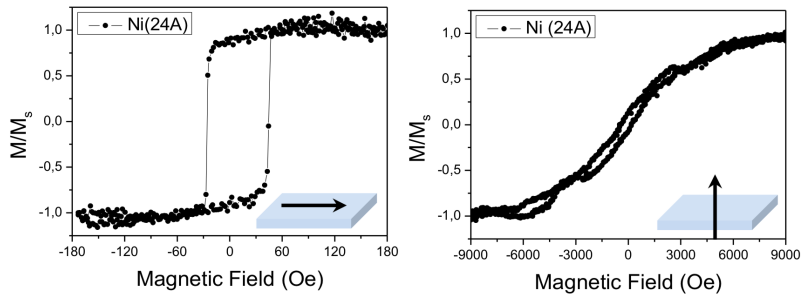

Magnetic Field $(\mathrm{Oe})$
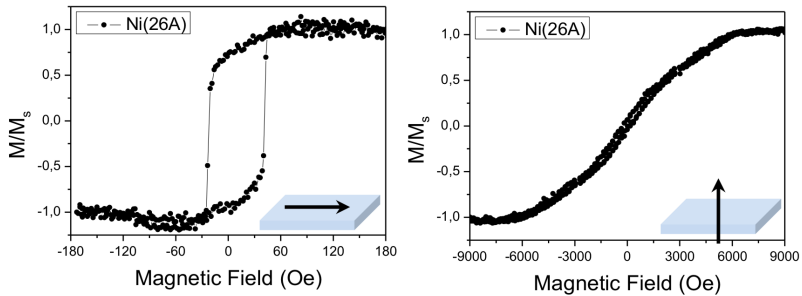

Fig. 3. Hysteresis loops of $\mathrm{Ni}$ thin films for different layer thicknesses. L-MOKE loops are shown on left, whereas P-MOKE loops are in the right column.

\section{Conclusions}

We have managed to prepare Ni films which show perpendicular magnetization on Si substrates. On the other hand, the easy axis switched from perpendicular to parallel direction with the increasing layer thickness. It is obviously possible to say that the thickness-driven, spin reorientation transition has been observed in Ni films deposited on Si substrates. 


\section{Acknowledgments}

The authors wish to acknowledge to Dr. Osman Öztürk for sample preparation system and Erdem Demirci for his kind help to perform MOKE measurements. This research was partially supported by TUBITAK through the project number 114F004.

\section{References}

[1] S. Iwasaki, K. Ouchi, IEEE Transactions on Magnetics 14(5), 849 (1978).

[2] M. Futamoto, N. Inaba, N. Hirayama, Y. Ito, Y. Honda, Journal of magnetism and magnetic materials 193(1), 36 (1999).
[3] S. Blügel, G. Bihlmayer, "Magnetism of LowDimensional Systems: Theory" in Handbook of Magnetism and Advanced Magnetic Materials, ed. by H. Kronmüller and S. Parkin, Wiley Online Library, 2007.

[4] B. Schulz, K. Baberschke, Phys. Rev. B 50, 13467 (1994).

[5] M. Yaqoob Khan, C.B. Wu, M. Erkovan, W. Kuch, J. Appl. Phys. 113, 023913 (2013).

[6] S. Tanuma, C.J. Powell, D.R. Penn, Surface and interface analysis 35, 268 (2003). 